\title{
Plasmonic biosensors and nanoprobes based on gold nanoshells
}

\author{
XU BeiBei, MA XiaoYuan, RAO YanYing, DONG Jian \& QIAN WeiPing* \\ State Key Laboratory of Bioelectronics, School of Biological Science and Medical Engineering, Southeast University, Nanjing 210096, China
}

Received March 4, 2011; accepted March 25, 2011

\begin{abstract}
Gold nanoshells (GNSs), consisting of a dielectric core coated with gold, have gained extensive attention as they show readily tunable optical properties and good biocompatibility. As highly sensitive and label-free optical biosensors with wide applications, GNSs have been investigated in many fields including drug delivery, immunoassay, cancer treatment, biological sensing and imaging. Taking advantage of the adjustability of the local surface plasmon resonance (LSPR) and the sensitivity of the surfaceenhanced Raman scattering (SERS) signal of GNSs, we have developed diverse applications including plasmonic biosensors and nanoprobes based on GNSs. In this review we introduce plasmonic and electromagnetic properties and fabrication methods of GNSs. We describe research progress in recent years, and highlight several applications of GNSs developed by our group. Finally we provide a brief assessment of future development of GNSs as plasmonic materials that can be integrated with complementary analytical techniques.
\end{abstract}

gold nanoshells, LSPR, SERS, 3D arrays, $\mathrm{H}_{2} \mathrm{O}_{2}$, antioxidant activity, biosensor, nanoprobe

Citation: $\quad$ Xu B B, Ma X Y, Rao Y Y, et al. Plasmonic biosensors and nanoprobes based on gold nanoshells. Chinese Sci Bull, 2011, 56: 3234-3241, doi: 10.1007/s11434-011-4670-1

With advances of technology, one-component materials can no longer meet the needs of society. Thus researchers have fabricated composite materials from two or more materials with complementary functions and optimization of performance [1-4]. In addition, nanomaterials of noble metals have gained widespread attention with their excellent physical and chemical properties and biocompatibility. Gold, as a typical representative of nanomaterials, has become the noble metal with the most dynamic research and development potential. Furthermore, novel core-shell structured nanomaterials with a variety of shapes, structures and/or controllable components have aroused intense interest in recent years. As a result, gold nanoshells (GNSs) have emerged as metal nanocomposite materials of great interest. GNSs containing a spherical dielectric core surrounded by an ultrathin, conductive gold layer not only offer superiority in performance but also great potential for developing simple, fast, and lowcost bioanalytical methods for biosensing and nanoprobes. As a new type of nanocomposite material, GNSs possess

*Corresponding author (email: wqian@ @ seu.edu.cn) many notable features. Their linear optical properties, infrared extinction characteristics, photothermal conversion properties, acoustic properties, cavity absorption and electron dynamics characteristics, make GNSs a focal point of basic and applied research. In addition to tunable LSPR among other features, GNSs show a strong electromagnetic enhancement effect, which makes GNSs a potentially ideal active SERS substrate $[5,6]$. Research in this area has revealed many new phenomena and raised many new issues, and provided new principles and new methods for nano-characterization technology and sensor technology, which will lead to new types of ultra-high sensitivity sensors for LSPR and SERS.

This paper will focus on the fabrication of GNSs and research progress in the field in chemical/biological sensing and surface-enhanced spectroscopy.

\section{Plasmonic and electromagnetic properties of GNSs}

The primary reason that GNSs have been of interest and 
studied intensively is their many features including linear optical properties, infrared extinction characteristics and luminescence properties. Nanoshells can be designed and fabricated with plasmon resonance tunable from the visible to the infrared regions of the spectrum, by varying the composition and dimensions of the layers of nanoparticles. The most common core materials for GNSs are polystyrene (PS) microspheres and silica $\left(\mathrm{SiO}_{2}\right)$ microspheres [7], both of which have advantages and disadvantages. The existing preparation methods can produce PS microspheres as small as $20 \mathrm{~nm}$ or as large as $5 \mu \mathrm{m}$, with high stability but poor uniformity [7-9]. $\mathrm{SiO}_{2}$ particles have good physical and chemical stability, and show little tendency to aggregate in colloidal solution. Furthermore, $\mathrm{SiO}_{2}$ has high optical permeability, and does not affect surface redox reactions [10]. More importantly, Stober et al. [11] reported in 1968 a method for preparation of highly uniform $\mathrm{SiO}_{2}$ particles with sizes from about $50 \mathrm{~nm}$ to $1 \mu \mathrm{m}$. For a given size of $\mathrm{SiO}_{2}$ core with gold shell, the LSPR of the nanoparticles, which determines the optical absorption and scattering of the particles, can be regulated by changing the thickness of the gold shell (adjusting the core-shell radius ratio, in fact) and the plasmon resonance red shifts from the visible to the infrared region, as shown in Figure 1 [12-15]. The aggregation state
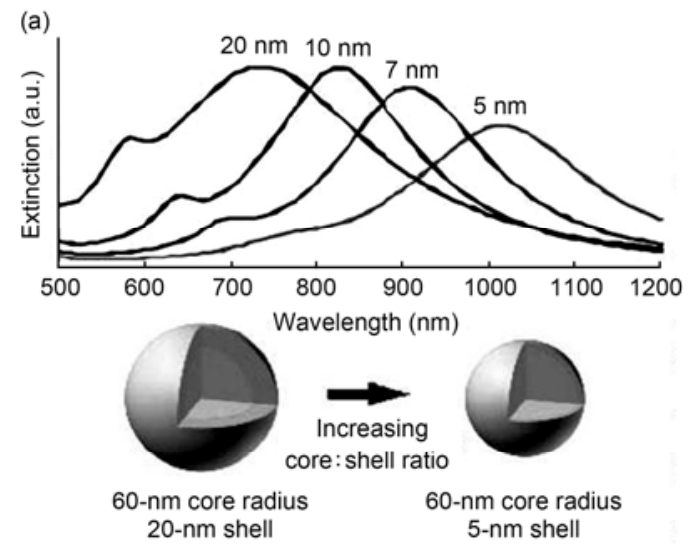

(b)
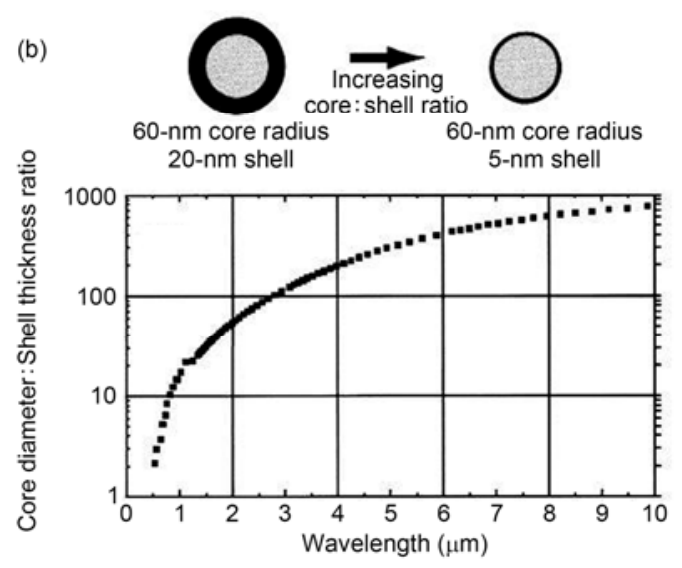

Figure 1 Optical tunability for nanoshells with $60-\mathrm{nm}$ silica core radius and gold shells 5, 7, 10 and $20 \mathrm{~nm}$ thick (a) [15]. The plasmon resonance of the particles red shifts with increasing core: shell radius ratio (b) [12]. of GNSs and variation of dielectric constant of their environment can also cause changes in the extinction spectrum [16-18].

Hao et al. [19] probed the microstructure of GNSs using TEM and demonstrated the existence of very small holes on the surface. By Mie scattering theory and the discrete dipole approximation method, the electron dynamics of the shell structure (including the complete shell, and a defective shell with holes) have been studied. The theoretical calculations showed that the 2-5 $\mathrm{nm}$ holes on the GNS surface had little effect on the extinction spectrum, but provided remarkable enhancement of electromagnetic field intensity. The intensity was found to increase 3- to 4-fold with peak field intensity increasing almost 10-fold. Prodan et al. [20] developed a simple electromagnetic simulation from molecular orbital theory, describing the plasmon resonance of complex nanostructures with arbitrary shape, which could explain the fundamental case of interaction or intercombination between plasmon resonances [21].

\section{Fabrication of GNSs with tunable plasmon resonance}

The many preparation methods of GNSs can be categorized as one-step methods and seed-mediated methods [22]. In one-step methods gold formed by reduction of $\mathrm{Au}(\mathrm{III}) \mathrm{di}-$ rectly deposits on the surface of $\mathrm{SiO}_{2}$ particles with or without amination to form GNSs [22,23]. In seed-mediated synthesis seeds, which may be small gold nanoparticles or other nanoparticles (e.g. nickel) are pre-formed on the surface of the $\mathrm{SiO}_{2}$ particles as deposition sites for gold produced by reduction [22,24]. The one-step method is relatively simple, but the surface of the prepared GNSs is rough with relatively poor controllability and reproducibility. Although the more complex seed-mediated process requires a longer time, the surface of the GNSs is much smoother with better controllability and repeatability. The preferred method for preparing uniformly sized GNSs is the seedmediated method developed by the Halas research group [12] at Rice University in the USA, and the Graf team [25] at Utrecht University in The Netherlands. Those self-assembly processes are the basis of the fabrication methods that have been developed by our group.

\subsection{Monodisperse GNSs in solution}

GNPs (gold nanoparticles), the precursors of GNSs, were fabricated as previously described [26] with some modifications. GNPs (2-5 $\mathrm{nm}$ in diameter), prepared by the reduction of $\mathrm{AuCl}_{4}^{-}$with $\mathrm{NaBH}_{4}$ as described elsewhere [27], were attached to (3-aminopropyl)triethoxy silane (APTES)functionalized silica colloids by electrostatic adsorption to form $\mathrm{SiO}_{2} / \mathrm{GNPs}$. The GNPs continue to grow via reduction of $\mathrm{AuCl}_{4}^{-}$to $\mathrm{Au}(0)$ with many reagents including formaldehyde 
[28], sodium borohydride, carbon monoxide gas and $\mathrm{H}_{2} \mathrm{O}_{2}$ [29], inducing the formation of GNSs as depicted in Figure 2.

Due to charge, the repulsive forces between $\mathrm{SiO}_{2} / \mathrm{GNPs}$ ensure stability in solution and the ability to be stored for long periods of time, and provide a stable foundation for subsequent experiments. In the growth of $\mathrm{SiO}_{2} / \mathrm{GNPs}$ into GNSs, the coverage of gold on the $\mathrm{SiO}_{2}$ surface increases with a raise of concentrations of $\mathrm{H}_{2} \mathrm{O}_{2}$ until a complete gold shell is formed. The LSPR of corresponding products is gradually redshifed throughout the shell-forming process. Subsequently, the growth of GNSs resulting from increased shell thickness induces a wavelength-dependent blueshift of the LSPR, in accordance with previous reports (Figures 3 and 4). Diverse applications developed by our group have been based mainly on the LSPR wavelength shift associated with the GNS growth process.

\subsection{Three-dimensional (3D) GNS arrays on solid surfaces}

We have also developed a facile, effective method for construction of 3D ordered macroporous (3DOM) matrixes by incorporation of gold nanoparticles (GNPs) with certain sizes into $3 \mathrm{D}_{\mathrm{SiO}_{2}}$ arrays prepared by vertical deposition.

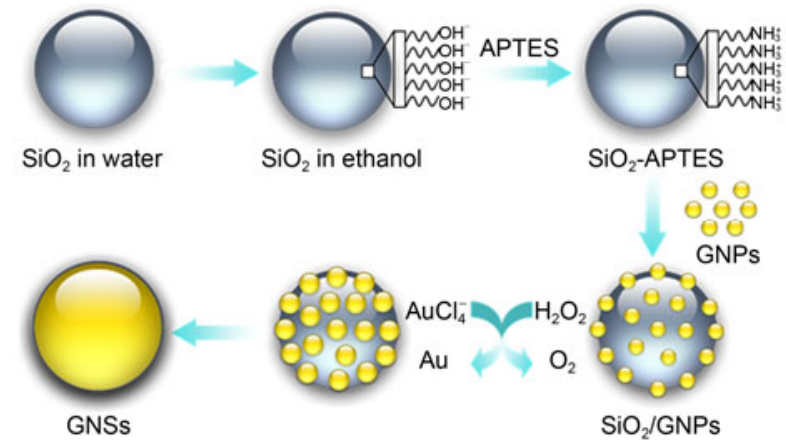

Figure 2 (Color online) Schematic illustration of the formation of $\mathrm{H}_{2} \mathrm{O}_{2}$ mediated GNSs [29].

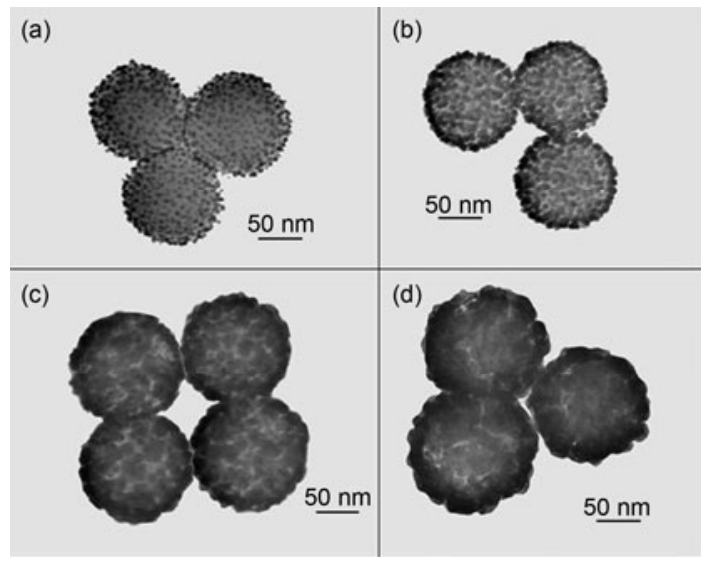

Figure 3 TEM images of (a) $\mathrm{SiO}_{2} / \mathrm{GNP}_{\mathrm{S}}$ upon reaction with varying concentrations of $\mathrm{H}_{2} \mathrm{O}_{2}$ from (b) 25 , (c) 100 , to (d) $200 \mu \mathrm{mol} / \mathrm{L}$ [29].
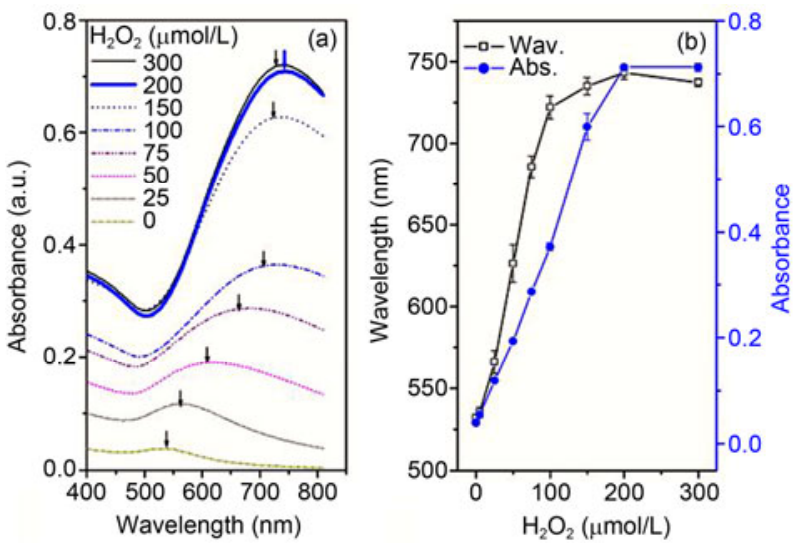

Figure 4 (Color online) (a) Absorption spectra for the growth of $\mathrm{SiO}_{2} / \mathrm{GNPs}$ in the presence of variable concentrations of $\mathrm{H}_{2} \mathrm{O}_{2}$ from 0 to $300 \mu \mathrm{mol} / \mathrm{L}$. (b) The calibration curve corresponding to the peak absorbance and wavelength of the reaction system with different concentrations of $\mathrm{H}_{2} \mathrm{O}_{2}$ [29].

The $3 \mathrm{D} \mathrm{SiO}_{2}$ arrays were then converted into self-supporting polystyrene (PS) macroporous films, which are depicted in Figure $5[30,31]$. To make 3D GNSs arrays play greater roles, such as an active SERS substrate, we recently developed self-assembled 3D ordered GNSs arrays by improved experimental methods [32].

The adsorption and subsequent growth of gold nanoparticles in the colloidal crystal was assessed by UV-vis absorption spectroscopy (Figure 6). Of the two distinct peaks that were observed, the absorption peak centered at $\sim 430$ $\mathrm{nm}$ is derived from the photonic band gap (PBG) produced by the ordered structure of $\mathrm{SiO}_{2}$ colloidal crystals. The peak at longer wavelength $(520 \mathrm{~nm})$ is attributed to the plasmon resonance of gold nanoparticles. Significant changes in the absorption spectrum occurred with increase of the number of reductions. The increased intensity of the LSPR of gold nanoparticles indicated a gradual increase of the amount of gold absorbed on $\mathrm{SiO}_{2}$ colloidal crystals; while the LSPR red shift was caused by the increased size of gold nanoparticles via reduction reaction. There was little or no variation in the position of the PBG absorption peak over the reduction cycles, showing that the original ordered structure was retained. Thus the reduction process (specifically the number of reductions) effectively controlled the size of the gold nanoparticles and did not disrupt the ordered structure of the $\mathrm{SiO}_{2}$ colloidal crystal. Using $\mathrm{SiO}_{2}$ colloidal crystals combined with gold nanoparticles of different sizes as templates, we have also prepared PS with ordered porous structure, with gold particles stably preserved in the wall structure of the PS. The GNP-infiltrated polystyrene (GNIPS) inverse opals retained the LSPR properties of GNPs, and duplicated the photonic features of the templates. Because of the reversible changes of the two characteristic peaks, these GNIPS films exhibited optical "on-off switching" capability, which was helpful in monitoring variations of the microenvironment originating from the permeation and release of surrounding media [31]. 
(a)

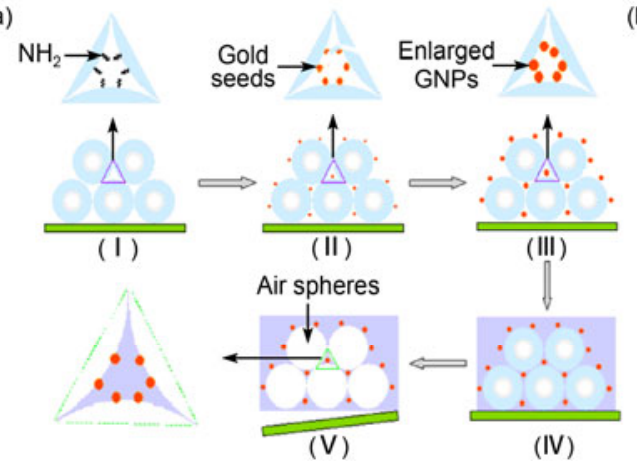

(b)

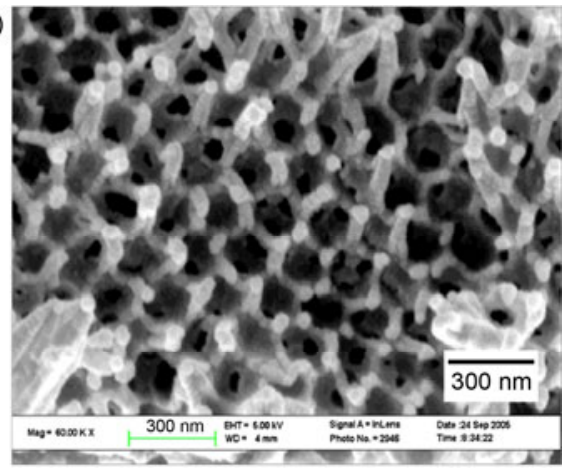

Figure 5 (Color online) Schematic illustration of the fabrication of GNP-embedded 3D matrixes (a); SEM image of a PS film embedded with gold seeds (b) [30].

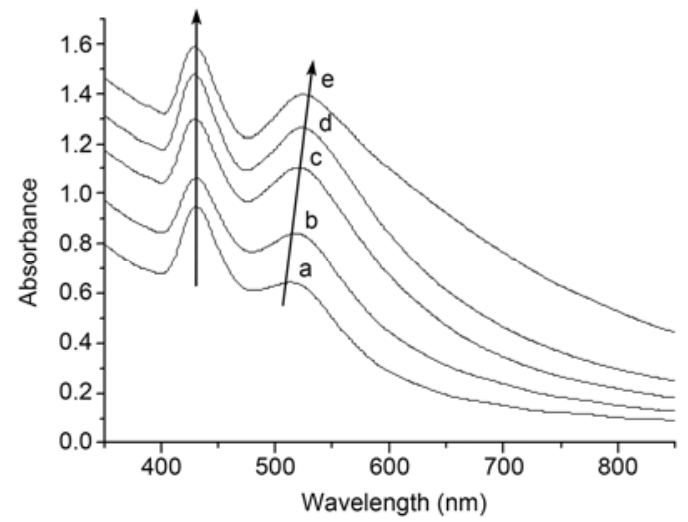

Figure 6 Absorption spectra recorded from gold-infiltrated silica templates during five cycles of $\mathrm{AuCl}_{4}^{-}$reduction process [30].

\section{Research progress of GNSs in our group}

\subsection{Label-free biosensors based on GNSs}

Khlebtsov et al. [33,34] achieved solid-phase dot immunoassay using GNSs. The method was based on staining nitrocellulose membrane (NCM) with GNSs modified with biological probe molecules. The results showed that the limit of detection was one order of magnitude lower than with gold particles, reaching $0.25 \mathrm{ng} / \mathrm{L}$. The limit of detection of this method was also closely related to the core-shell ratio of GNSs. In 2010, Xia et al. [35] detected human IgG antigen by displacement of the absorption peak with PScored GNSs modified by the goat anti-human IgG antibody.

At an early stage of our research we investigated the formaldehyde-mediated growth process of GNSs, and obtained GNSs with infrared extinction properties [28]. GNSs have potential biological applications in view of the permeability of whole blood to near-infrared radiation. Self-assembled monolayers (SAMs) of GNSs as label-free biosensors were developed for monitoring biomolecular interactions in diluted whole blood, as illustrated in Figure 7 [36]. GNSs with controllable shell thickness were self-assembled on silanized glass by electrostatic adsorption. We investigated the LSPR sensitivity of SAMs to changes of refractive index (RI), and demonstrated that the SAMs can be used to monitor the adsorption of bovine serum albumin (BSA) in real time based on the changes in the absorption spectrum.

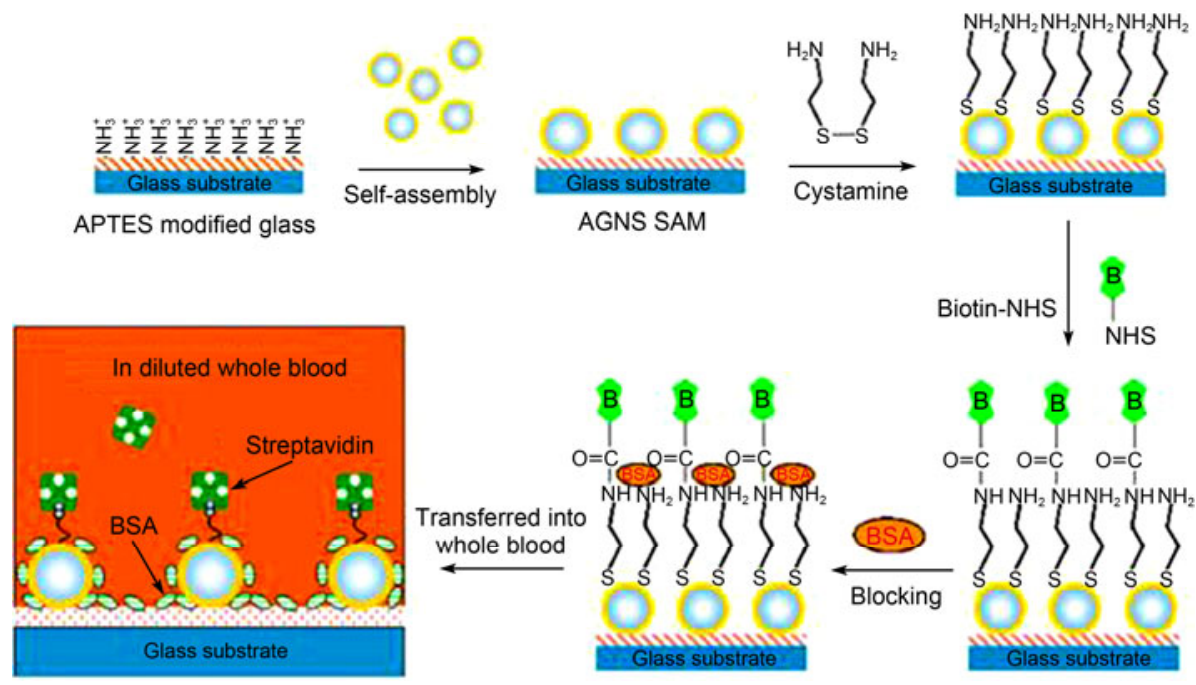

Figure 7 (Color online) Schematic fabrication process of GNS SAMs and analysis of streptavidin [36]. 
Furthermore, the SAMs were modified with biotin via covalent bonding, and fabricated as a biosensor for streptavidin. The SAMs of GNSs were developed as a novel, simple and low-cost optical biosensor with short assay time, requiring no sample purification or separation, with detection limit $\sim 3 \mu \mathrm{g} / \mathrm{mL}$ and a wide dynamic range of $3-50 \mu \mathrm{g} / \mathrm{mL}$ of streptavidin.

A novel nanocomposite-based method for the detection of L-DOPA and tyrosinase (TR) activity was reported this year [37]. We first constructed growth-sensitive GNS precursor nanocomposites $\left(\mathrm{SiO}_{2} / \mathrm{GNPs}\right.$ II). Owing to reduction of $\mathrm{AuCl}_{4}^{-}$to $\mathrm{Au}(0)$ by L-DOPA, $\mathrm{SiO}_{2} / \mathrm{GNPs}$ II became larger and grew into GNSs with changes in LSPR wavelength that correlated well with the concentration of $L$-DOPA. Since $L$-DOPA is formed by TR-catalyzed hydroxylation of $L$-tyrosine, this method can also be used to determine the activity of TR (Figure 8), which is of importance in clinical work and the food industrial sector.

\subsection{Antioxidant function evaluation based on GNSs nanoprobes}

$\mathrm{H}_{2} \mathrm{O}_{2}$ is not only a commonly used chemical reagent, but also an important reactive oxygen species in biological systems. As $\mathrm{H}_{2} \mathrm{O}_{2}$ has generally been considered as a damaging entity, by mediating pathogenic processes, related tests have much physiological significance. Based on the $\mathrm{H}_{2} \mathrm{O}_{2}$-mediated formation process of GNSs, a novel nanocomposite-based method for achieving concentration detection of $\mathrm{H}_{2} \mathrm{O}_{2}$ and estimating antioxidant activity of a series of reagents was developed by our group. In view of the good correlation of LSPR wavelength shift with $\mathrm{H}_{2} \mathrm{O}_{2}$ concentration (Figure 3), $\mathrm{H}_{2} \mathrm{O}_{2}$ scavenging activities of several antioxidants were determined by inhibiting the $\mathrm{H}_{2} \mathrm{O}_{2}$-mediated formation of GNSs from $\mathrm{SiO}_{2} /$ GNPs [29]. To compare the hydrogen peroxide scavenging activity $\left(\mathrm{SA}_{\mathrm{HP}}\right)$ of different antioxidants, the following formula was used to calculate inhibition rate

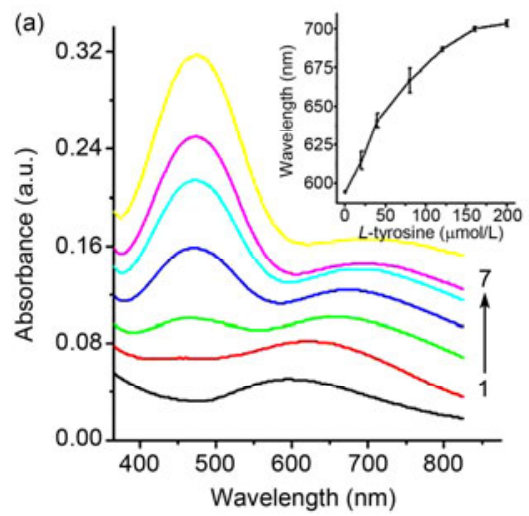

of the wavelength: $I_{w}=\Delta w / \Delta w_{0}(\%)$. The meaning of $\Delta w$ and $\Delta w_{0}$ is shown on the left of Figure 9. The color changes coincident with the LSPR wavelength shifts could be observed visually in the $\mathrm{H}_{2} \mathrm{O}_{2}$-mediated formation process of GNSs because of intervention of varying concentrations of antioxidative tannic acid as shown on the right of Figure 9. In the determination process for antioxidant capacity, the LSPR spectra were in the visible region, in the range $530-750 \mathrm{~nm}$. Thus the color changes of the solution could be observed by the naked eye without the need for a spectrophotometer, which provided the possibility of visual colorimetry for qualitative or quantitative analysis of antioxidant capacity. Compared with traditional methods (such as fluorescence radiation and chromatography), this method has great potential practical value in rapid screening analysis of antioxidants.

$\mathrm{SA}_{\mathrm{HP}}$ of a series of phenolic acids were tested on similar principles, with several tea and herb extracts also evaluated [38]. As an original detection strategy, we combined nanoscience with food and health research of great significance. The spectral changes caused by variation of $\mathrm{H}_{2} \mathrm{O}_{2}$ concentration could be monitored not only by UV-vis-near infrared spectrophotometry $[29,38]$, but also by Raman spectrometry. We studied the relationship between the surface structure of the growing GNSs, optical properties and SERS activity. During the growth process, the SERS activity of the $\mathrm{SiO}_{2} /$ GNPs with different enlargement conditions clearly changed with increasing coverage of $\mathrm{SiO}_{2}$ cores by gold, which was quantitatively correlated with the concentration of $\mathrm{H}_{2} \mathrm{O}_{2}$ used (Figure 10). GNSs were used as SERS probes to develop a new method for the evaluation of antioxidant capacity with detection limits down to $\sim 5 \mu \mathrm{mol} / \mathrm{L}$, illustrating the advantages of SERS for biological sample analysis [39].

\subsection{D GNS arrays as SERS nanoprobes}

SERS has emerged as a new powerful analytical technique

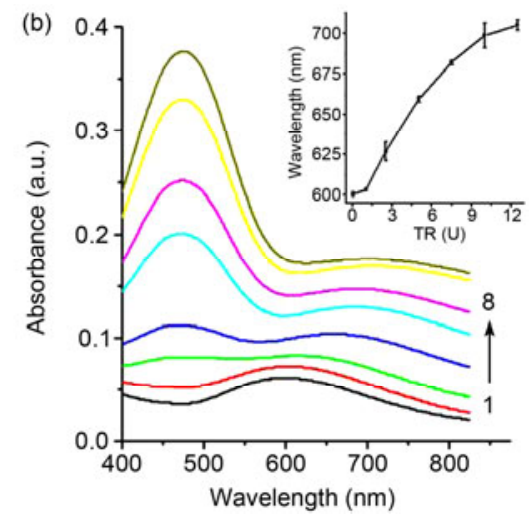

Figure 8 (Color online) (a) Absorption spectra corresponding to the TR-stimulated growth of GNSs in the presence of variable concentrations of $L$-tyrosine: (1) 0, (2) 20, (3) 40, (4) 80, (5) 120, (6) 160, and (7) $200 \mu \mathrm{mol} / \mathrm{L}$. Inset: Calibration curve for the optical resonance wavelength of the resulting GNSs at different concentrations of $L$-tyrosine. (b) Absorption spectra of GNSs grown by variable concentrations of TR: (1) 0, (2) 1, (3) 2.5, (4) 5, (5) 7.5, (6) 10, (7) 12.5, and (8) 15 U. Inset: Calibration curve for the optical resonance wavelength of the resulting GNSs at different concentrations of TR [37]. 

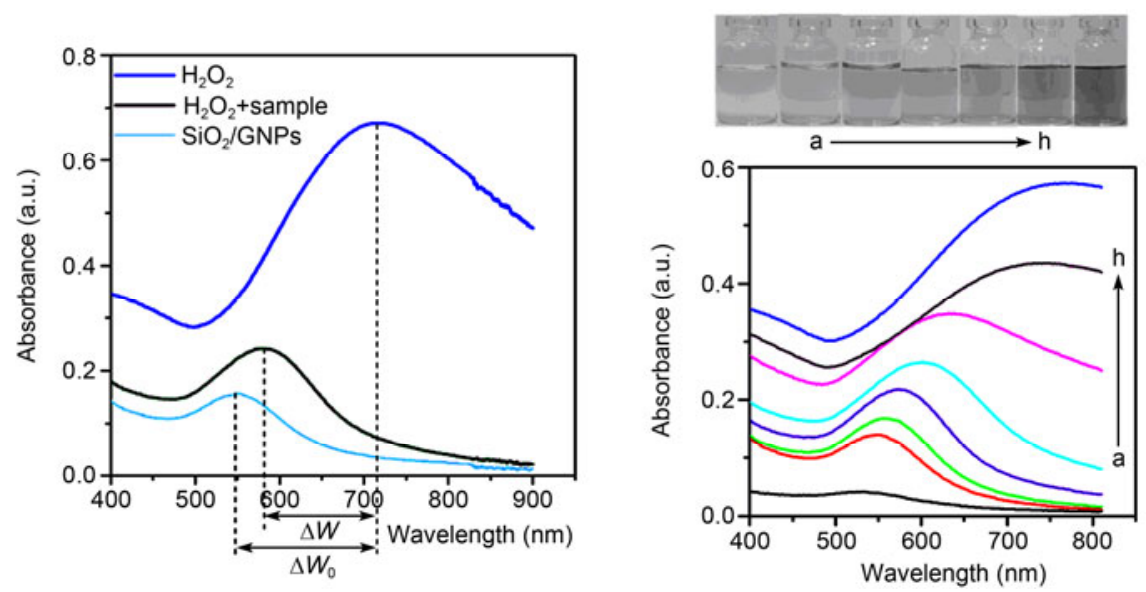

Figure 9 (Color online) Schematic diagram of the method for expressing $\mathrm{H}_{2} \mathrm{O}_{2}$ scavenging activity (left); Images of the color change and the corresponding $\mathrm{UV}$-vis spectra for the reaction system in the presence of varying concentration of tannic acid. $\mathrm{SiO}_{2} / \mathrm{GNPs}$ (a) upon reaction with $200 \mu \mathrm{mol} / \mathrm{L} \mathrm{H}_{2} \mathrm{O}_{2}$ and tannic acid with concentration from $200 \mu \mathrm{mol} / \mathrm{L}$ (b) to $0 \mu \mathrm{mol} / \mathrm{L}$ (h) (right) [29].
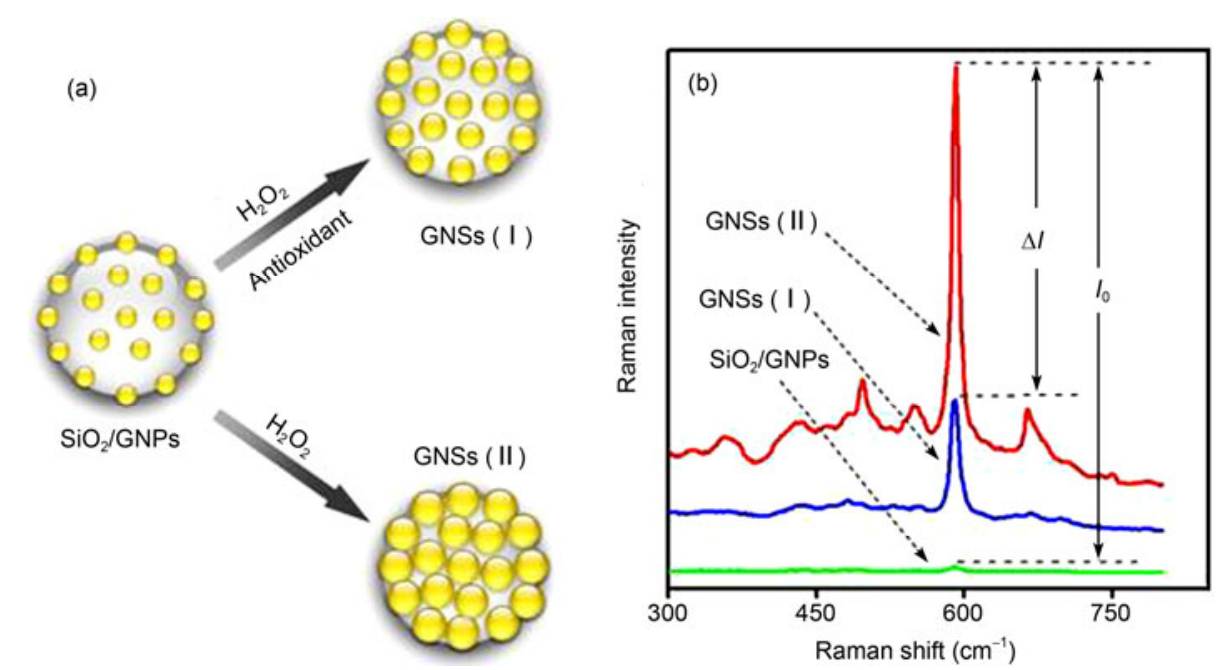

Figure 10 (Color online) Schematic diagram of the methodology for detecting $\mathrm{H}_{2} \mathrm{O}_{2}$ scavenging activity. (a) GNSs produced with [GNSs (I)] or without [GNSs (II)] antioxidant. (b) SERS spectra of the Raman probe molecule using different substrates: $\mathrm{SiO}_{2} / \mathrm{GNPs}$ (green trace), GNSs (I) (blue) and GNSs (II) (red) [39].

for detecting and identifying molecules with ultrahigh sensitivity, on the basis of their unique vibrational energy levels. SERS gives high signal enhancement and fluorescence quenching effects as well as "whole fingerprint" information, and low water background, which are extremely desirable for biological sample analysis. Two principal enhancement mechanisms are generally considered to explain the SERS phenomenon, namely long-range electromagnetic enhancement factors and short-range chemical enhancement factors [40]. The electromagnetic enhancement, which is thought to contribute most $\left(\sim 10^{4}-10^{7}\right)$ to the observed intensity enhancement in SERS, results from Raman excitation and emission coupling with LSPR modes of nanoparticles. Because the LSPR can be easily tuned by changing the size and shape of the nanoparticles as well as the space between them, LSPR sensors are ideal candidates for a complementary molecular identification platform with SERS. A great deal of research has yet to be translated into the development of an ideal SERS substrate for practical applications. The ideal substrate should produce high enhancement, give a reproducible and uniform response, have a long shelf-life, and be easily fabricated and sufficiently simple for integration into analytical devices. Recent studies show that highly ordered metal nanostructured arrays can provide an excellent platform for this purpose.

Halas and coworkers [41] obtained GNS arrays after evaporation of the solvent, by modifing GNSs with cetyltrimethylammonium bromide (CTAB) and dropwise addition onto a silicon substrate. They found that the SERS enhancement effect of the substrate was about 10 to 20 times as strong by this method as that of the single GNS. Moreover, the GNS array substrates integrated SERS and SEIRA on a single substrate by enhancing both RS and IRA spectroscopy. Abdelsalam et al. [42] achieved detection of a flavin 
analog with sub-femtomolar limit of detection using colloidal templates to produce Ag patterns of inverse hexagonal arrays for electrochemical SERS. Recent developments have resulted in substrates with large areas patterned on the nanoscale, as demonstrated by the work of Cai et al. [43]. They first prepared polystyrene (PS) colloidal ordered array by spin-coating, then deposited a 3-nm thick silver film on the PS microspheres by sputtering. As the cathode, the substrate was used to obtain two-dimensional ordered arrays of silver nanoshells by depositing silver nanoparticles on the surface using electrophoresis, and tunable SERS and LSPR features were both studied. Despite the successes of SERS research, the potential applications of SERS-activity changes of plasmonic substrates are often neglected. In pioneering work of our group 3D ordered GNSs have been used to assess $\mathrm{H}_{2} \mathrm{O}_{2}$ scavenging activity via the changes of SERSactivity at different forming stages of $3 \mathrm{D}$ ordered core-shell nanostructured arrays [32] as shown in Figure 11. The 3D GNS arrays, with high sensitivity, uniformity and repeatability of SERS signal, can be a simple and subtle platform for assessing $\mathrm{H}_{2} \mathrm{O}_{2}$ scavenging activity, multivariate analysis and so on.
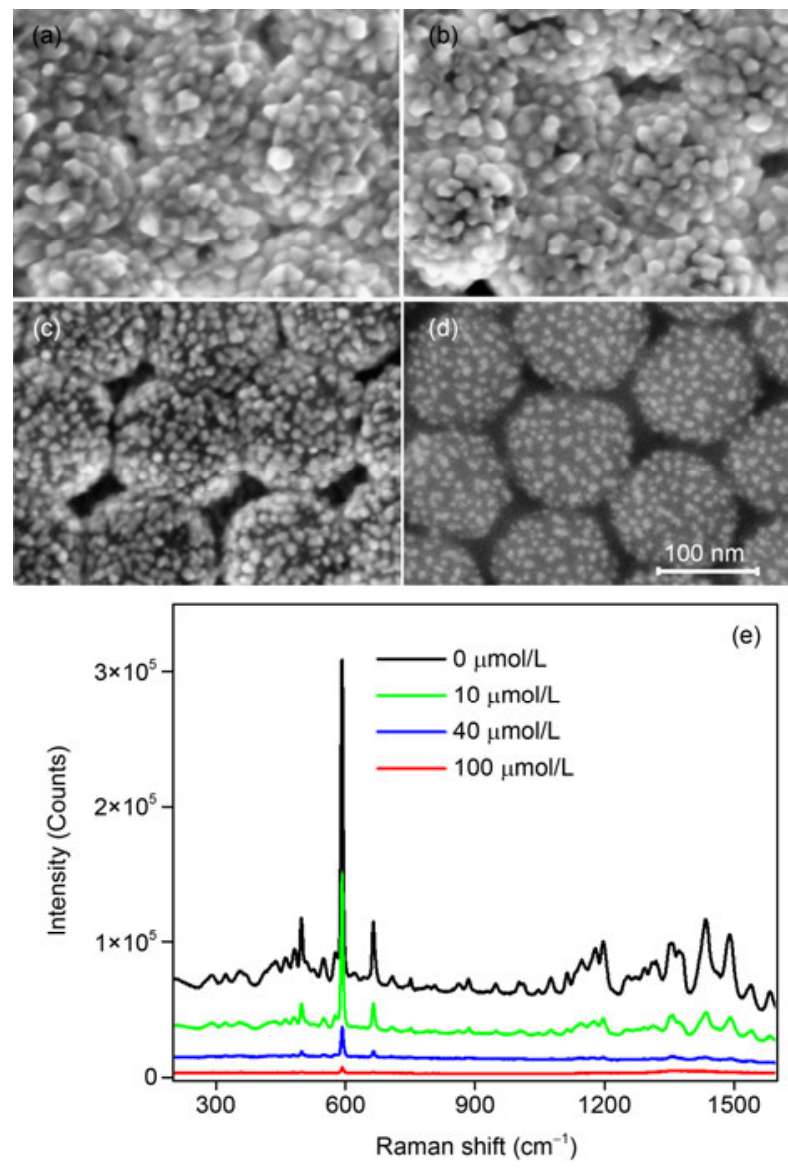

Figure 11 (Color online) SEM and relevant SERS spectra of NBA of $\mathrm{SiO}_{2} / \mathrm{GNPs}$ arrays upon reaction with $200 \mu \mathrm{mol} / \mathrm{L} \mathrm{H}_{2} \mathrm{O}_{2}$ and different concentrations of tannic acid: (a) 0, (b) 10, (c) 40, (d) $100 \mu \mathrm{mol} / \mathrm{L}$. (e) SERS spectra of $10^{-6} \mathrm{~mol} / \mathrm{L}$ NBA on GNSs array substrates that were produced with $200 \mu \mathrm{mol} / \mathrm{L} \mathrm{H}_{2} \mathrm{O}_{2}$ and a series of tannic acid concentrations [32].

\section{Summary and outlook}

We have constructed homogeneous substrates of GNSs, and 2D and 3D GNS arrays as detection and assay platforms. Based on the LSPR wavelength shift in the chemical-mediated formation process of GNSs from GNPs, we achieved success in monitoring and detecting diverse molecules, or interaction between them. GNSs possess many features, making possible broad application prospects in many areas. For example, as active SERS substrates GNSs can be used in research on single molecules, biomedical science, the food and drug industry, and environmental monitoring. The strong optical extinction features of GNSs provide effective access to the near-infrared region $(700-1300 \mathrm{~nm})$, which is extremely useful for many biomedical applications. On the one hand, optical transmission through human tissue is optimal in this wavelength range; on the other hand, the surface of GNSs can be modified to easily combine with biological systems. Thus traditional biological recognition and optical properties of GNSs can be skillfully combined through biological coupling. Compared to traditional colloidal gold, GNSs have more optical controllability and higher chemical stability.

Although much work has been performed on the development of LSPR-shift assay and related study of molecular identification techniques with GNSs, many important challenges stand in the way of efficient use in practice, including improvements in detection capability and sensitivity, combination with other practical or advanced techniques, etc. To sum up, GNSs should truly be an up-and-coming contender for analytical devices for biological, biophysical and biochemical studies with crossover research effort to extract and conjoin all of these features for developing highly practical, sensitive and reliable platforms for use as biosensors and analysis devices.

This work was supported by the National Natural Science Foundation of China (90923010), the National Basic Research Program of China (2010CB933902) and the Promoting Project for Industrialization of Scientific Research Achievement of Regular Institutions of Higher Education in Jiangsu Province (2009-34).

1 Montazeri M, Fickenscher M, Smith L M, et al. Direct measure of strain and electronic structure in $\mathrm{GaAs} / \mathrm{GaP}$ core-shell nanowires. Nano Lett, 2010, 10: 880-886

2 Gole A, Stone J W, Gemmill W R, et al. Iron oxide coated gold nanorods: Synthesis, characterization, and magnetic manipulation. Langmuir, 2008, 24: 6232-6237

3 Wang Y Q, Nikitin K, McComb D W. Fabrication of $\mathrm{Au}-\mathrm{Cu}_{2} \mathrm{O}$ core-shell nanocube heterostructures. Chem Phys Lett, 2008, 456: 202-205

4 Wang H, Brandl D W, Le F, et al. Nanorice: A hybrid plasmonic nanostructure. Nano Lett, 2006, 6: 827-832

5 Kerker M, Blatchford C G. Elastic-scattering, absorption, and surface-enhanced raman-scattering by concentric spheres comprised of a metallic and a dielectric region. Phys Rev B, 1982, 26: 4052-4064

6 Talley C E, Jackson J B, Oubre C, et al. Surface-enhanced Raman 
scattering from individual Au nanoparticles and nanoparticle dimer substrates. Nano Lett, 2005, 5: 1569-1574

7 Kalele S, Gosavi S W, Urban J, et al. Nanoshell particles: Synthesis, properties and applications. Curr Sci, 2006, 91: 1038-1052

8 He G W, Pan Q M. Synthesis of polystyrene and poly styrene/ poly(methyl methacrylate) nanoparticles. Macromol Rapid Commun, 2004, 25: 1545-1548

9 Kim J H, Chainey M, Elaasser M S, et al. Preparation of highly sulfonated polystyrene model colloids. J Polym Sci A Polym Chem, 1989, 27: 3187-3199

10 Ung T, Liz-Marzan L M, Mulvaney P. Controlled method for silica coating of silver colloids. Influence of coating on the rate of chemical reactions. Langmuir, 1998, 14: 3740-3748

11 Stober W, Fink A, Bohn E. Controlled growth of monodisperse silica spheres in the micron size range. J Colloid Interface Sci, 1968, 26: 62-69

12 Oldenburg S J, Averitt R D, Westcott S L, et al. Nanoengineering of optical resonances. Chem Phys Lett, 1998, 288: 243-247

13 Oldenburg S J, Jackson J B, Westcott S L, et al. Infrared extinction properties of gold nanoshells. Appl Phys Lett, 1999, 75: 2897-2899

14 Stewart M E, Anderton C R, Thompson L B, et al. Nanostructured plasmonic sensors. Chem Rev, 2008, 108: 494-521

15 Hirsch L R, Gobin A M, Lowery A R, et al. Metal nanoshells. Ann Biomed Eng, 2006, 34: 15-22

16 Oldenburg S J, Hale G D, Jackson J B, et al. Light scattering from dipole and quadrupole nanoshell antennas. Appl Phys Lett, 1999, 75: 1063-1065

17 Pissuwan D, Valenzuela S M, Cortie M B. Therapeutic possibilities of plasmonically heated gold nanoparticles. Trend Biotechnol, 2006, 24: 62-67

18 Moghimi S M, Hunter A C, Murray J C. Nanomedicine: Current status and future prospects. FASEB J, 2005, 19: 311-330

19 Hao E, Li S Y, Bailey R C, et al. Optical properties of metal nanoshells. J Phys Chem B, 2004, 108: 1224-1229

20 Prodan E, Radloff C, Halas N J, et al. A hybridization model for the plasmon response of complex nanostructures. Science, 2003, 302: 419-422

21 Radloff C, Halas N J. Plasmonic properties of concentric nanoshells. Nano Lett, 2004, 4: 1323-1327

22 Phonthammachai N, Kah J C Y, Jun G, et al. Synthesis of contiguous silica-gold core-shell structures: Critical parameters and processes. Langmuir, 2008, 24: 5109-5112

23 Phonthammachai N, White T J. One-step synthesis of highly dispersed gold nanocrystals on silica spheres. Langmuir, 2007, 23: 11421-11424

24 Lim Y T, Park O O, Jung H T. Gold nanolayer-encapsulated silica particles synthesized by surface seeding and shell growing method: Near infrared responsive materials. J Colloid Interface Sci, 2003, 263: $449-453$

25 Graf C, van Blaaderen A. Metallodielectric colloidal core-shell particles for photonic applications. Langmuir, 2002, 18: 524-534

26 Leff D V, Brandt L, Heath J R. Synthesis and characterization of hydrophobic, organically-soluble gold nanocrystals functionalized with primary amines. Langmuir, 1996, 12: 4723-4730
27 Phadtare S, Kumar A, Vinod V P, et al. Direct assembly of gold nanoparticle "shells" on polyurethane microsphere "cores" and their application as enzyme immobilization templates. Chem Mater, 2003, 15: $1944-1949$

28 Tan Y, Ding S H, Qian W P, et al. Fabrication of gold nanoshells and their potential applications to biology (in Chinese). Acta Chim Sinica, 2005, 63: 929-933

29 Li H, Dong J, Qian W P, et al. Development of methodology based on the formation process of gold nanoshells for detecting hydrogen peroxide scavenging activity. Anal Chem, 2009, 81: 8916-8922

30 Ding S H, Qian W P, Tan Y, et al. In situ incorporation of gold nanoparticles of desired sizes into three-dimensional macroporous matrixes. Langmuir, 2006, 22: 7105-7108

31 Tan Y, Qian W P, Ding S H, et al. Gold-Nanoparticle-infiltrated polystyrene inverse opals: A three-dimensional platform for generating combined optical properties. Chem Mater, 2006, 18: 3385-3389

32 Rao Y Y, Chen Q F, Qian W P, et al. Growth-sensitive 3D ordered gold nanoshells precursor composite arrays as SERS nanoprobes for assessing hydrogen peroxide scavenging activity. Analyst, 2011, 136: 769-774

33 Khlebtsov B, Dykman L, Khlebtsov N, et al. A solid-phase dot assay using silica/gold nanoshells. Nanoscale Res Lett, 2007, 2: 6-11

34 Khlebtsov B, Khlebtsov N. Enhanced solid-phase immunoassay using gold nanoshells: Effect of nanoparticle optical properties. Nano, 2008, 19: 435703

35 Xia Y T, Lu W S, Jiang L. Fabrication of color changeable polystyrene spheres decorated by gold nanoparticles and their label-free biosensing. Nano, 2010, 21: 085501

36 Wang Y, Qian W P, Tan Y, et al. A label-free biosensor based on gold nanoshell monolayers for monitoring biomolecular interactions in diluted whole blood. Biosens Bioelectron, 2008, 23: 1166-1170

37 Kong F J, Liu H F, Qian W P, et al. Growth-sensitive gold nanoshells precursor nanocomposites for the detection of L-DOPA and tyrosinase activity. Biosens Bioelectron, 2011, 26: 1902-1907

38 Ma X Y, Li H, Qian W P, et al. Determination of hydrogen peroxide scavenging activity of phenolic acids by employing gold nanoshells precursor composites as nanoprobes. Food Chem, 2011, 126: 698-704

39 Chen Q F, Rao Y Y, Qian W P, et al. Raman spectroscopy for hydrogen peroxide scavenging activity assay using gold nanoshell precursor nanocomposites as SERS probes. Anal Methods, 2011, 3: 274-279

40 Zhao L, Jensen L, Schatz G C. Pyridine-Ag 20 cluster: A model system for studying surface-enhanced Raman scattering. J Am Chem Soc, 2006, 128: 2911-2919

41 Wang H, Kundu J, Halas N J. Plasmonic nanoshell arrays combine surface-enhanced vibrational spectroscopies on a single substrate. Angew Chem, 2007, 119: 9198-9202

42 Abdelsalam M, Bartlett P N, Russell A E, et al. Quantitative electrochemical SERS of flavin at a structured silver surface. Langmuir, 2008, 24: 7018-7023

43 Yang S K, Cai W P, Lei Y, et al. Surface nanometer-scale patterning in realizing large-scale ordered arrays of metallic nanoshells with well-defined structures and controllable properties. Adv Funct Mater, 2010, 20: $1-7$

Open Access This article is distributed under the terms of the Creative Commons Attribution License which permits any use, distribution, and reproduction in any medium, provided the original author(s) and source are credited. 